\title{
NEAR-INFRARED PHOTOMETRIC STUDY OF THE OLD OPEN CLUSTER TRUMPLER 5
}

\author{
Sang Chul Kim, Jaemann Kyeong, and Eon-Chang Sung \\ Korea Astronomy and Space Science Institute, Daejeon 305-348, Korea \\ E-mail: sckim, jman, ecsung@kasi.re.kr \\ (Received November 30, 2009; Accepted December 21, 2009)
}

\begin{abstract}
We present JHK near-infrared photometric study for the old open cluster (OC) Trumpler 5 (Tr 5 ), based on the 2MASS data. From the color-magnitude diagrams of Tr 5 , we have located the position of the red giant clump (RGC) stars, and used the mean magnitude of the RGC stars in $K$-band to estimate the distance to $\operatorname{Tr} 5, \mathrm{~d}=3.1 \pm 0.1 \mathrm{kpc}\left((m-M)_{0}=12.46 \pm 0.04\right)$. From fitting the theoretical isochrones of Padova group, we have estimated the reddening, metallicity, and age : $E(B-V)=0.64 \pm 0.05,[\mathrm{Fe} / \mathrm{H}]$ $=-0.4 \pm 0.1 \mathrm{dex}$, and $t=2.8 \pm 0.2 \mathrm{Gyr}(\log t=9.45 \pm 0.04)$, respectively. These parameters generally agree well with those obtained from the previous studies on $\operatorname{Tr} 5$ and confirms that this cluster is an old OC with metallicity being metal-poorer than solar abundance, located in the anti-Galactic center region.
\end{abstract}

Key words : open clusters and associations: individual (Trumpler 5) - Galaxy: disk - Galaxy: stellar content - Galaxy: structure - Hertzsprung-Russell diagram

\section{INTRODUCTION}

The recent update of the Galactic open cluster (OC) catalogue by Dias et al. (2002) provides 1787 OCs (version 2.10, 2009 February 17), while there exist many reports with smaller number of new OCs, such as Bica, Dutra, \& Barbuy (2003), Kharchenko et al. (2005) and Froebrich, Scholz, \& Raftery (2007; "FSR objects") (see also, Lada \& Lada 2003; Kim 2006, and references therein). Kronberger et al. (2006) discovered 66 stellar groupings from an inspection of the DSS (Digitized Sky Survey) and 2MASS (Two Micron All Sky Survey ${ }^{1}$, Skrutskie et al. 1997, 2006) images of selected Milky Way regions, whose morphologies, color-magnitude diagrams (CMDs), and stellar density distributions suggest that these objects are possible OCs. Paunzen \& Netopil (2006) have characterized the current status on the accuracy of parameters for 395 OCs with more than three independent measurements from the literature, and suggested 72 standard OCs with the most accurate known parameters which could be served as a standard table in the future for testing isochrones and stellar models.

Old OCs, with ages greater than $\sim 1$ Gyr, are an important tool for the study of the formation and the evolution of the Galactic disk (Friel 1995; Chen, Hou, \& Wang 2003). Trumpler 5 (Tr 5; C0634+094, OCL 494, Lund 237, Collinder 105) is one of the old OCs located in anti-Galactic center region with very small galactic

$0 \quad$ Corresponding Author: S. C. Kim

1 available at http://www.ipac.caltech.edu/2mass/releases/ allsky/ latitude (Kim \& Sung 2003, and references therein) and among the most massive OCs in the Galaxy (Kaluzny 1998; Cole et al. 2004). The coordinates of Tr 5 are $\alpha_{J 2000}=06^{h} 36^{m} 42.0^{s}, \delta_{J 2000}=+09^{\circ} 25^{\prime} 58.8^{\prime \prime}, l=$ $202 .^{\circ} 87$, and $b=+01 .^{\circ} 05$ (Lyngå 1987). The Trumpler class of $\operatorname{Tr} 5$ is III $1 \mathrm{r}$, which means (i) $\operatorname{Tr} 5$ is detached and shows no concentration, (ii) most stars in this cluster are of nearly the same brightness, and (iii) it is rich. Table 1 shows the compilation of the previous estimates of the fundamental parameters of Trumpler 5. The studies of Kaluzny (1998), Kim \& Sung (2003), and Piatti, Clariá, \& Ahumada (2004) are the only studies based on CCD observations. Recently, Cole et al. (2004) and Carrera et al. (2007) have used the Ca II triplet $(8498,8542$, and $8662 \AA)$ line strengths to measure the metallicity of $\operatorname{Tr} 5$.

$\operatorname{Tr} 5$ is an OC located very close to the Galactic plane $\left(b=+01 .{ }^{\circ} 05\right)$. Therefore, as Table 1 shows, the mean interstellar reddening toward $\operatorname{Tr} 5$ is $E(B-V)=0.6$, which gives optical extinction of $A_{V} \sim 1.9 \mathrm{mag}$. In the near-infrared (NIR) band, this extinction decreases much to $A_{J}=0.28 A_{V}=0.5 \mathrm{mag}$ and $A_{K}=0.11 A_{V}=$ $0.2 \mathrm{mag}$ (Cardelli, Clayton, \& Mathis 1989). $\operatorname{Tr} 5$ is also known to have differential reddenings (Piatti et al. 2004). NIR photometric study, therefore, is a better way to study this cluster than the studies using optical photometry data. In this paper, we have analyzed the NIR photometry data of $\operatorname{Tr} 5$ obtained from the 2MASS project.

Section II describes the NIR data sets used in this study and Sections III presents the CMDs of $\operatorname{Tr} 5$. Sections IV, V, and VI present the distance, Padova 


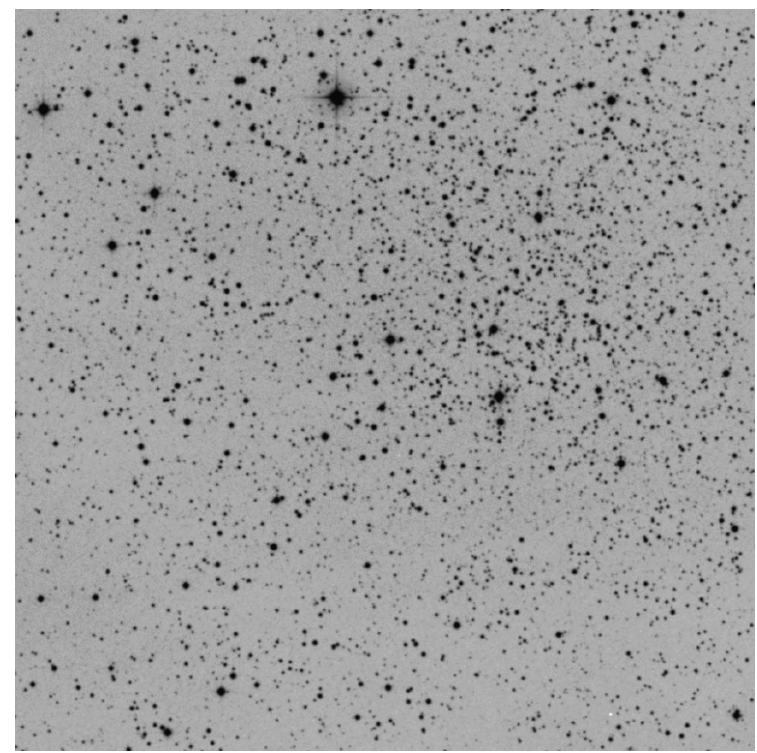

Fig. 1. - Second generation DSS optical red image of the cluster $\operatorname{Tr} 5$. The field of view is $15^{\prime} \times 15^{\prime}$. North is up and east is to the left. The coordinate of the cluster center is $\left(\alpha_{J 2000}, \delta_{J 2000}\right)=\left(06^{h} 36^{m} 42.0^{s},+09^{\circ} 25^{\prime} 58.8^{\prime \prime}\right)$.

isochrone fitting results, and the color-color diagram, respectively, for the $\mathrm{OC} \operatorname{Tr} 5$. Finally, a summary is given in Section VII.

\section{THE 2MASS DATA}

The 2MASS project (Skrutskie et al. 2006) have used two dedicated $1.3 \mathrm{~m}$ telescopes located at Mount Hopkins, Arizona, and Cerro Tololo, Chile and $256 \times$ 256 NICMOS3 (HgCdTe) arrays manufactured by Rockwell International Science Center (now Rockwell Scientific), which give field-of-view of $8 .^{\prime} 5 \times 8 .^{\prime} 5$ and pixel scale of $2^{\prime \prime}$ pixel $^{-1}$ (Kim 2006). The photometric system comprise $J(1.25 \mu \mathrm{m}), H(1.65 \mu \mathrm{m})$ and $K_{S}(2.16$ $\mu \mathrm{m})$ bands, where the " $K$-short" $\left(K_{S}\right)$ filter excludes wavelengths longward of $2.31 \mu \mathrm{m}$ to reduce thermal background and airglow and includes wavelengths as short as $2.00 \mu \mathrm{m}$ to maximize bandwidth (see Figure 2 of Skrutskie et al. 2006 or Figure 7 of Bonatto, Bica, \& Girardi 2004 for the transmission curves of the 2MASS filters; Carpenter 2001).

VizieR $^{2}$ was used to extract $J, H$, and $K_{S}$ 2MASS photometry data in circular areas centered on the cluster $\operatorname{Tr} 5$. Figure 1 displays the grey-scale image of $\operatorname{Tr} 5$ taken from the DSS. 2MASS $J, H$, and $K_{S}$-band images covering the field of $\operatorname{Tr} 5$ are shown in Figures 2, 3 , and 4 , respectively.

The distribution of photometric errors is shown in Figure 5. 2MASS photometric errors typically attain $0.1 \mathrm{mag}$ at $J \approx 16.0 \mathrm{mag}, H \approx 15.4 \mathrm{mag}$, and $K_{S} \approx 14.6$ mag. These photometric uncertainties are

${ }^{2}$ http://vizier.u-strasbg.fr/viz-bin/VizieR?-source=2MASS

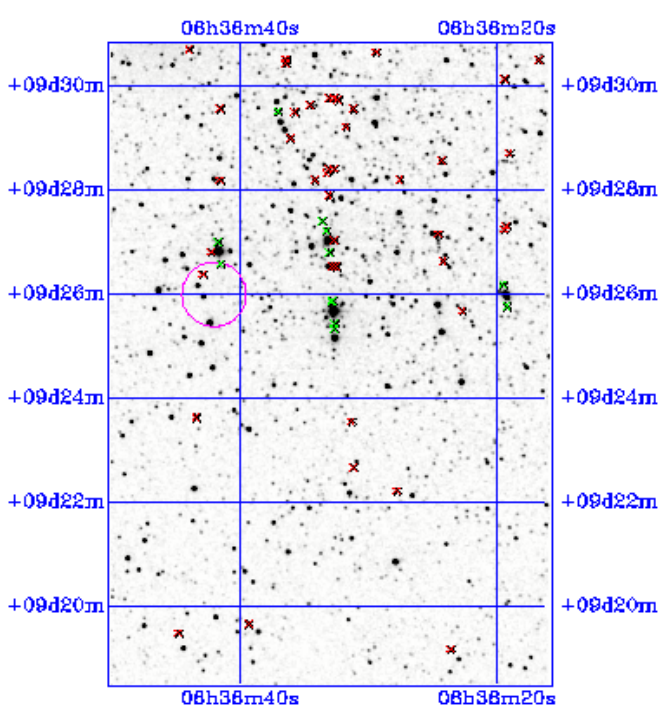

Fig. 2.- 2MASS $J$-band image of the cluster $\operatorname{Tr} 5$, provided by the 2MASS Image Service. The open circle indicates the object central part. Crosses indicate instrumental artifacts.

of high enough quality, comparable to other NIR photometric studies (see, e.g., Bonatto, Bica, \& Santos (2005), Bonatto \& Bica (2007); see also Sung, Stauffer, \& Bessell (2009) for the distribution of photometric errors of the Spitzer Space Telescope Infrared Array Camera (IRAC) undersampled data).

\section{THE COLOR-MAGNITUDE DIAGRAMS}

Figure 6 shows the $J$ vs. $(J-H)$ (panel a) and $K_{S}$ vs $\left(J-K_{S}\right)$ (panel b) CMDs for the $\operatorname{Tr} 5$ stars in radial region of $\mathrm{R}<7.7^{\prime}$. From the stellar density profile of $\operatorname{Tr} 5$ stars observed with the Cerro Tololo Inter-American Observatory (CTIO) $0.9 \mathrm{~m}$ telescope, Piatti et al. (2004) have measured the angular radius of $\operatorname{Tr} 5$ as $\mathrm{R} \sim 7.7^{\prime} \pm 0.3^{\prime}$, which is used to plot Figure 6 . Thick band of many stars are seen below the mainsequence turn-off (MSTO). Broad red giant branch $(\mathrm{RGB})$ and red giant clump $\left(\mathrm{RGC} ; J_{R G C}=11.9 \pm 0.1\right.$, $K_{S, R G C}=11.0 \pm 0.1,(J-H)_{R G C}=0.73 \pm 0.03$, and $\left.\left(J-K_{S}\right)_{R G C}=0.90 \pm 0.05\right)$ can also be found.

Several stars are located at the blue straggler (BS) region above the MSTO and bluer than $J-H \approx 0.5$ and $J-K_{S} \approx 0.65$. Since $\operatorname{Tr} 5$ is located at the Galactic plane $\left(b=+01 .^{\circ} 05\right)$, it is needed to get detailed membership studies for $\operatorname{Tr} 5$ based either on radial velocity or proper motion to make a robust BS list with as least as possible field star contamination (Ahumada \& Lapasset 1995; De Marchi et al. 2006; Ahumada \& Lapasset 2007; Carraro, Vázquez, \& Moitinho 2008; Kyeong et al. 2008; Momany et al. 2008). We have statistically tested the possible number of BS stars in the Tr 5 field by comparing the CMDs of $\operatorname{Tr} 5$ and two nearby control fields. Two control fields are chosen at 
TABLE 1.

A List of the Estimation of the Physical Parameters of Trumpler 5

\begin{tabular}{|c|c|c|}
\hline Parameter & Information & Reference \\
\hline \multirow[t]{9}{*}{ Reddening, $E(B-V)$} & $0.80 \mathrm{mag}$ & Dow \& Hawarden 1970; Janes \& Adler 1982 \\
\hline & $0.48 \mathrm{mag}$ & Kalinowski 1974; Kalinowski et al. 1974 \\
\hline & $0.60 \mathrm{mag}$ & Piccirillo et al. 1977 \\
\hline & $0.64 \mathrm{mag}$ & Kalinowski 1979; Janes \& Adler 1982 \\
\hline & $0.58 \mathrm{mag}$ & Kaluzny 1998 \\
\hline & $0.60 \pm 0.10 \mathrm{mag}$ & Kim \& Sung 2003 \\
\hline & $0.66 \mathrm{mag}$ & Cole et al. 2004 \\
\hline & $0.60 \pm 0.04 \mathrm{mag}$ & Piatti et al. 2004 \\
\hline & $0.64 \pm 0.05 \mathrm{mag}$ & This study \\
\hline mean & $0.62 \pm 0.08 \mathrm{mag}$ & \\
\hline \multirow[t]{11}{*}{ Distance, $\mathrm{d}$} & $2.4 \mathrm{kpc}\left((m-M)_{0}=11.92 \mathrm{mag}\right)$ & Dow \& Hawarden 1970; Janes \& Adler 1982 \\
\hline & $2.9 \pm 0.1 \mathrm{kpc}\left((m-M)_{0}=12.3 \pm 0.1 \mathrm{mag}\right)$ & Kalinowski 1974; Kalinowski et al. 1974 \\
\hline & $2.4 \pm 0.3 \mathrm{kpc}\left((m-M)_{0}=11.9 \pm 0.3 \mathrm{mag}\right)$ & Kalinowski 1975 \\
\hline & $1.9 \mathrm{kpc}\left((m-M)_{0}=11.4 \mathrm{mag}\right)$ & Piccirillo et al. 1977 \\
\hline & $1.0 \mathrm{kpc}\left((m-M)_{0}=10.02 \mathrm{mag}\right)$ & Kalinowski 1979; Janes \& Adler 1982 \\
\hline & $3.0 \mathrm{kpc}\left((m-M)_{0}=12.4 \mathrm{mag}\right)$ & Kaluzny 1998 \\
\hline & $3.4 \pm 0.3 \mathrm{kpc}\left((m-M)_{0}=12.64 \pm 0.20 \mathrm{mag}\right)$ & Kim \& Sung 2003 \\
\hline & $2.8 \mathrm{kpc}$ & Cole et al. 2004 \\
\hline & $2.4 \pm 0.5 \mathrm{kpc}\left((m-M)_{0}=11.88 \pm 0.30 \mathrm{mag}\right)$ & Piatti et al. 2004 \\
\hline & $3.1 \pm 0.1 \mathrm{kpc}\left((m-M)_{0}=12.46 \pm 0.04 \mathrm{mag}\right)$ & This study \\
\hline & $2.5 \pm 0.7 \mathrm{kpc}\left((m-M)_{0}=11.9 \pm 0.8 \mathrm{mag}\right)$ & \\
\hline \multirow[t]{7}{*}{ Age } & 4.9 Gyr (from the morphological age index) & Janes \& Phelps 1994; Cole et al. 2004 \\
\hline & 4.1 Gyr & Kaluzny 1998 \\
\hline & $2.4 \pm 0.2$ Gyr $(\log t=9.38 \pm 0.04)$ & Kim \& Sung 2003 \\
\hline & $5.67 \pm 2.26 \mathrm{Gyr}$ & Salaris et al. 2004 \\
\hline & $3.0 \pm 0.5 \mathrm{Gyr}$ & Cole et al. 2004 \\
\hline & $5.0 \pm 0.5 \mathrm{Gyr}$ & Piatti et al. 2004 \\
\hline & $2.8 \pm 0.2$ Gyr $(\log t=9.45 \pm 0.04)$ & This study \\
\hline mean & $4.0 \pm 1.2$ Gyr $(\log t=9.60 \pm 0.15)$ & \\
\hline \multirow[t]{7}{*}{ Metallicity, $[\mathrm{Fe} / \mathrm{H}]$} & 0.0 dex & Kaluzny 1998 \\
\hline & $-0.30 \pm 0.10 \mathrm{dex}$ & Kim \& Sung 2003 \\
\hline & $-0.56 \pm 0.11 \mathrm{dex}^{\dagger}$ & Cole et al. 2004 \\
\hline & $-0.30 \pm 0.15 \mathrm{dex}$ & Piatti et al. 2004 \\
\hline & $-0.36 \pm 0.05 \mathrm{dex}^{\ddagger}$ & Carrera et al. 2007 \\
\hline & $-0.4 \pm 0.1 \mathrm{dex}$ & This study \\
\hline & $-0.32 \pm 0.17 \mathrm{dex}$ & \\
\hline
\end{tabular}

$\dagger$ : From Ca II triplet lines of 10 RGB member stars

$\ddagger$ : From Ca II triplet lines of 21 member stars

the same Galactic latitude, but with one degree larger and one degree smaller Galactic longitude for control field 1 and control field 2, respectively, than that of $\mathrm{Tr}$ 5. From the comparison of the CMDs of Tr 5 and the control fields for a given magnitude and color range, we counted the number of stars in a control field and subtracted this number of stars in the Tr 5 CMD randomly.

In Figure 7, we showed the raw CMD of $\mathrm{R}<6^{\prime}$ field of $\operatorname{Tr} 5$ (panel (a)), two CMDs of the control fields 1 and 2 (panels (b) and (c), respectively), and the field star eliminated CMDs using the control fields 1 and 2 (panels (d) and (e), respectively). The solid boxes in Figure 7 are plotted to estimate the number of stars in the BS region. There are 29 stars in the box of Fig.
7 (a), 20 and 23 stars in those of Fig. 7 (b) and (c), respectively, and 14 and 12 stars remain in those of Fig. 7 (d) and (e), respectively, after the elimination of the field star contamination. This indicates that $\gtrsim 40$ $\%$ stars in the BS region of Tr 5 CMD possibly could be bona-fide BSs, although the number of stars in the BS region increases at outer regions than the center (Figures 8 and 9).

From the wide width of the MS and the tilted placement of the RGC (following the reddening vector) in the CMDs of Tr 5, Piatti et al. (2004) have shown that $\operatorname{Tr} 5$ has non-uniform extinctions $[\Delta E(B-V)=$ $0.11-0.22]$ over the face of the cluster. Piatti et al. (2004) showed that the stars with relatively small photometric errors and/or the lowest color excesses are 


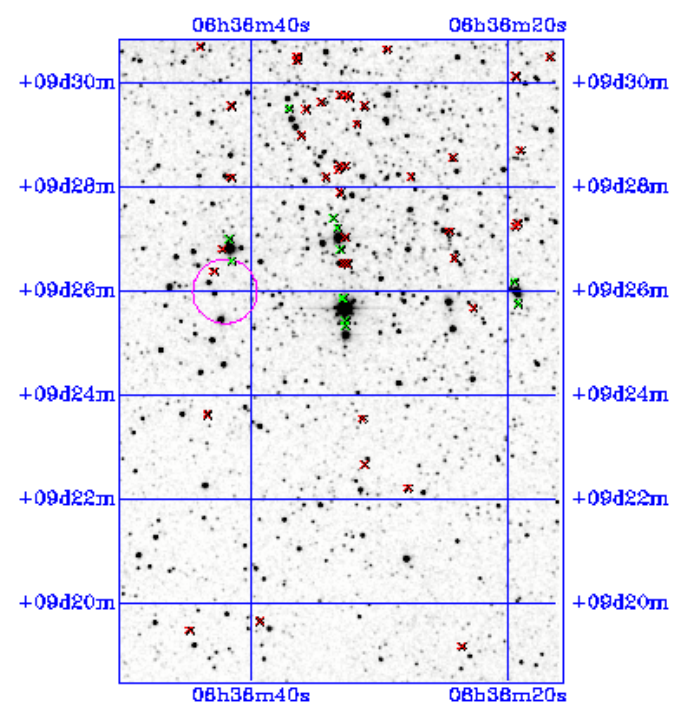

Fig. 3.- 2 MASS $H$-band image of the cluster $\operatorname{Tr} 5$, provided by the 2MASS Image Service. The open circle indicates the object central part. Crosses indicate instrumental artifacts.

mostly distributed in the cluster core region $\left(\mathrm{R}<2^{\prime}\right)$ and in the northeastern half of their observed field. They also showed that the highest reddened group of stars is found towards the south and there is an obscure cloud of interstellar matter as large as a quarter of ring of $\sim 2^{\prime}$ wide at $3^{\prime}$ from the cluster center towards the southwestern side. Figure 6 (a) clearly reveals that the RGC is tilted and extended and the RGB has wide width. These facts reveal the existence of the differential reddening in the field of $\operatorname{Tr} 5$, while it is not so clear in the CMD of $K_{S}$ vs $\left(J-K_{S}\right)$ (Figure $6(\mathrm{~b})$ ) which passband is less affected by the reddenings.

\section{DISTANCE}

The helium-burning RGC stars are known to have great potential to be used as a standard candle and there are claims that their absolute magnitude in a certain band is constant or that the luminosity of RGC is a function of age and/or metal abundance (see, e.g., Grocholski \& Sarajedini 2002). Janes \& Phelps (1994) estimated the mean color and magnitude of the RGC in old open clusters to be $(B-V)_{0, R G C}=0.95 \pm 0.10$, and $M_{V, R G C}=0.90 \pm 0.40$, when the $V$ magnitude difference between the RGC and the MSTO of the clusters, $\delta V$, is greater than one. Using Hipparcos RGC stars with parallax errors of less than $10 \%$ to calculate the $I$-band absolute magnitude of the solar neighborhood RGC, Paczyński \& Stanek (1998) find $M_{I, R G C}=$ $-0.28 \pm 0.09 \mathrm{mag}$ having no variation with color over the range $0.8<(V-I)_{0}<1.4$, and Stanek \& Garnavich (1998) find a similar result with $M_{I, R G C}=-0.23 \pm 0.03$ mag. From the $K$-band luminosity of 238 RGC stars, Alves (2000) finds $M_{K, R G C}=-1.61 \pm 0.03$ mag with

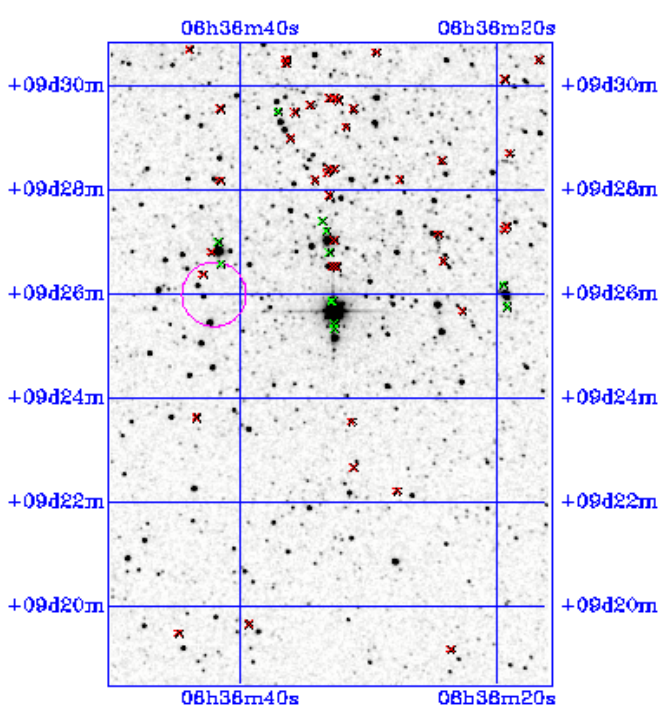

Fig. 4.- 2 MASS $K_{S}$-band image of the cluster $\operatorname{Tr} 5$, provided by the 2MASS Image Service. The open circle indicates the object central part. Crosses indicate instrumental artifacts.
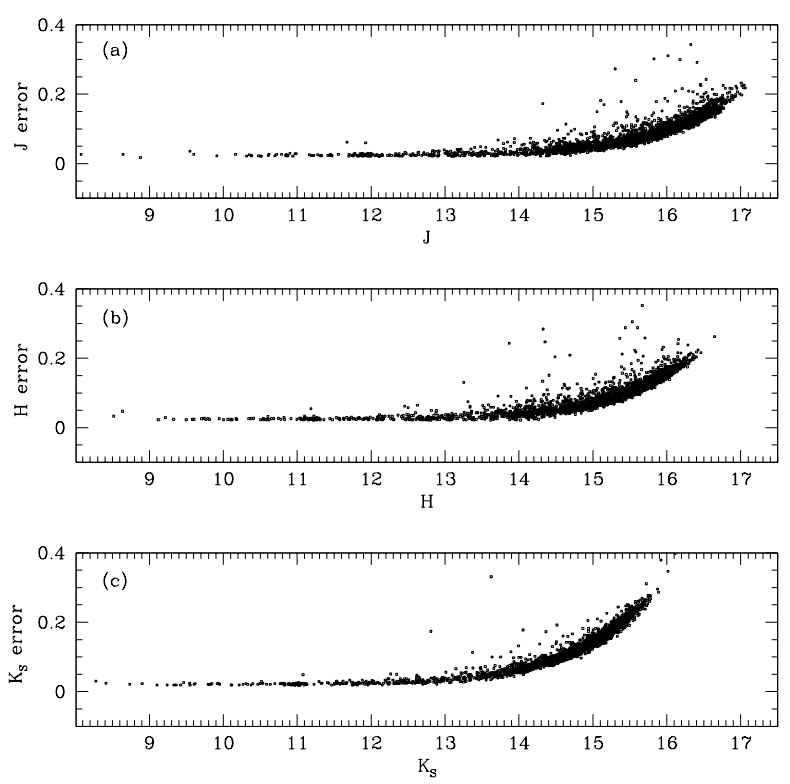

Fig. 5.- Distribution of the $J, H$, and $K_{S}$-band photometric errors as a function of magnitude.

no correlation between $[\mathrm{Fe} / \mathrm{H}]$ and $M_{K}$, and Grocholski \& Sarajedini (2002) find $M_{K, R G C}=-1.62 \pm 0.06$ mag from the $J K$ photometry of 14 OCs. The other advantage that the $K$-band holds is the relatively small amount of reddening effect in this band, therefore making it relatively free of the differential reddening effect (seen noticeably by the tilt of the RGC stars in the optical CMDs in Kim \& Sung (2003) and $J$ vs. $(J-H)$ CMDs in Figure 8) and efficient in determining the 

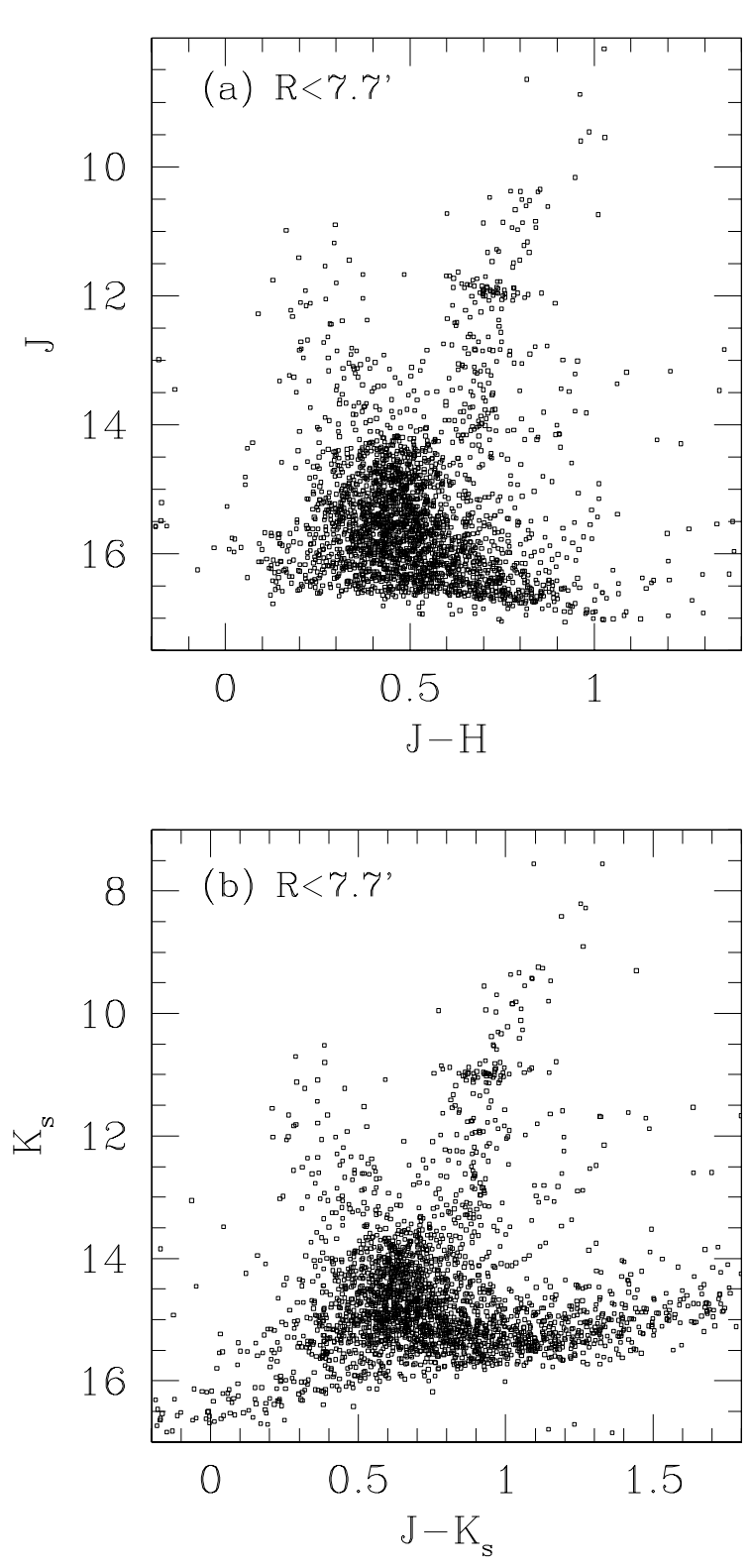

Fig. 6. - (a) $J$ vs. $(J-H)$ and (b) $K_{S}$ vs $\left(J-K_{S}\right)$ CMDs for the stars in the field $\left(\mathrm{R}<7.7^{\prime}\right)$ of $\operatorname{Tr} 5$.

mean magnitude of the RGC stars.

If we convert the 2MASS $K_{S}$-band to the $K$-band adopting the Bessell \& Brett (1988) system using the transformation equation A1 of Carpenter (2001),

$$
K_{B B}=K_{S}-(-0.044 \pm 0.003)-(0.000 \pm 0.005)\left(J-K_{B B}\right),
$$

we get the magnitude of the RGC obtained in the previous section to be $K_{R G C}=11.04 \pm 0.06 \mathrm{mag}$. If we adopt the absolute magnitude of the RGC stars of Gro-

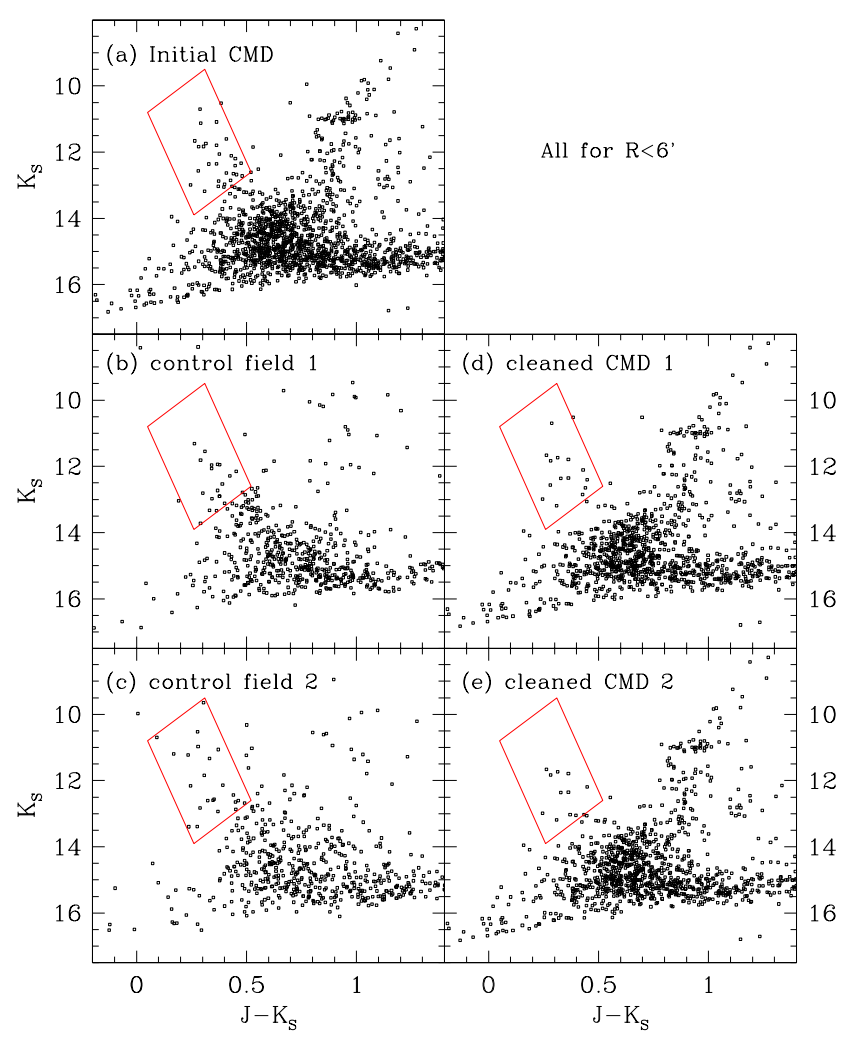

Fig. 7. - Statistical elimination of field star contamination. (a) $K_{S}$ vs. $\left(J-K_{S}\right)$ CMDs for the stars in the $\mathrm{R}<6^{\prime}$ field of $\operatorname{Tr} 5$. (b) and (c) are CMDs for the control fields 1 and 2 (all for $\mathrm{R}<6^{\prime}$ ), respectively, and (d) and (e) are field star contamination eliminated CMDs using the control fields 1 and 2, respectively. The solid boxes are plotted to estimate the number of stars in the BS region.

cholski \& Sarajedini (2002), $M_{K, R G C}=-1.62 \pm 0.06$ mag, we get the apparent distance modulus of $\operatorname{Tr} 5$, $\left(K-M_{K}\right)_{R G C}=12.66 \pm 0.04 \mathrm{mag}$ and the true distance modulus of $(m-M)_{0}=\left(K-M_{K}\right)_{R G C}-A_{K}=12.46 \pm$ $0.04 \mathrm{mag}(\mathrm{d}=3.1 \pm 0.1 \mathrm{kpc})$, using $A_{K}=0.11 A_{V}$.

\section{PADOVA ISOCHRONE FITTING}

In Figure 8, we have plotted the $J$ vs. $(J-H)$ CMDs for the Tr 5 stars in different radial regions as indicated in each panel from (a) $\mathrm{R}<2^{\prime}$ to (f) $\mathrm{R}<7.7^{\prime}$. We have fitted the theoretical Padova isochrones computed with the 2MASS $J, H$ and $K_{S}$ filters (Bonatto, Bica, \& Girardi 2004; Bica, Bonatto, \& Blumberg 2006) to derive the cluster parameters (Kim 2006). As Table 1 shows that the $E(B-V)$ reddening values toward $\operatorname{Tr} 5$ obtained from the previous studies (especially the recent studies) converge to $\sim 0.6 \mathrm{mag}$, we also obtained the best fit using this reddening value of $E(B-V)=0.60 \pm$ $0.10 \mathrm{mag}$ in Figure 8 (using $E(J-H)=0.309 E(B-V)$, 


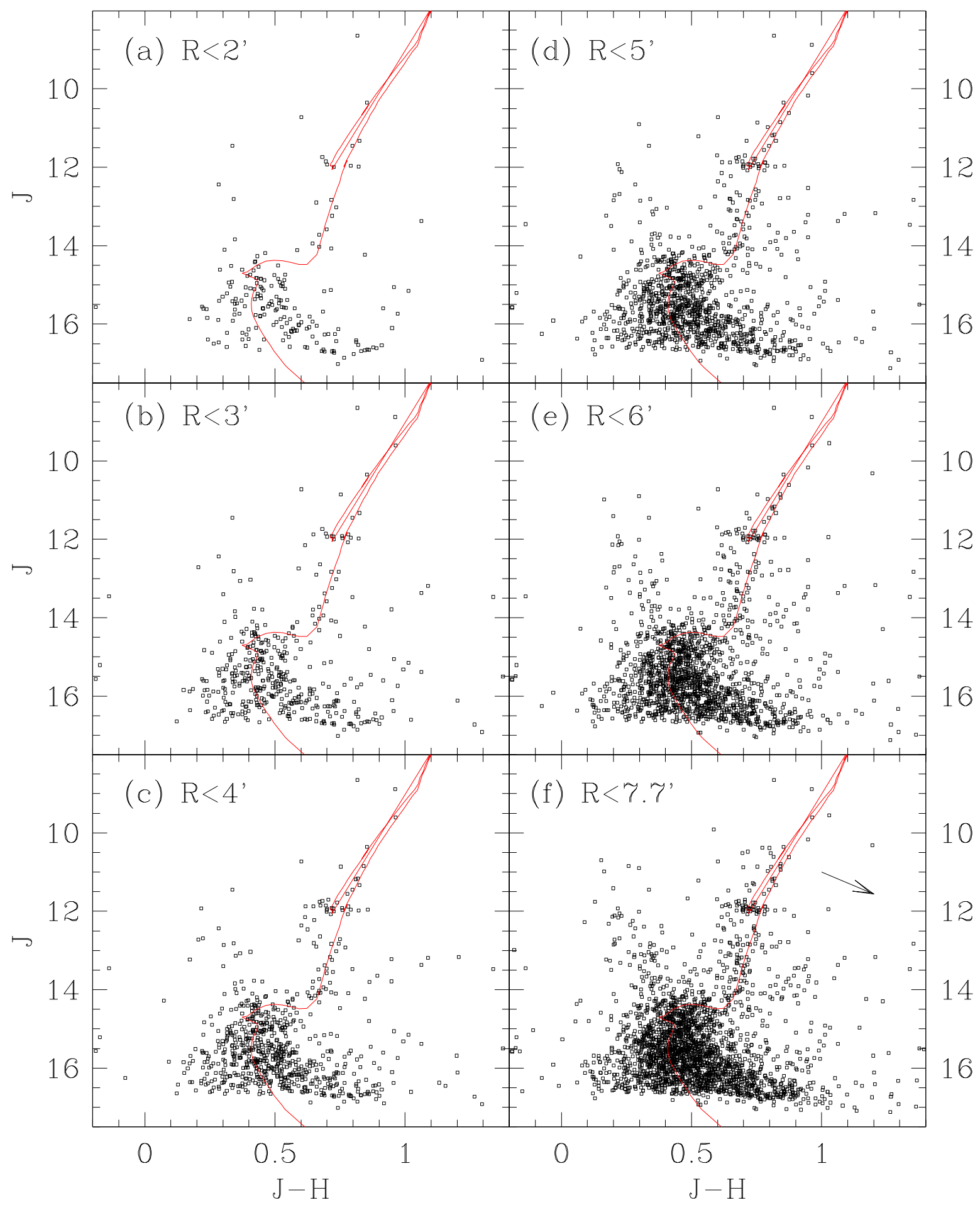

Fig. 8. - $J$ vs. $(J-H)$ CMDs for the stars in the field of $\operatorname{Tr} 5$. The solid lines represent the Padova isochrones (Bonatto, Bica, \& Girardi 2004) for $E(B-V)=0.60, J-M_{J}=12.98, \mathrm{Z}=0.008$, and age $=2.8$ Gyr. The reddening vector with $E(J-H)=0.20$ is shown in panel (f), where $A_{J}=0.28 A_{V}, R_{V}=3.1$ and $E(J-H)=0.309 E(B-V)$ are used. 


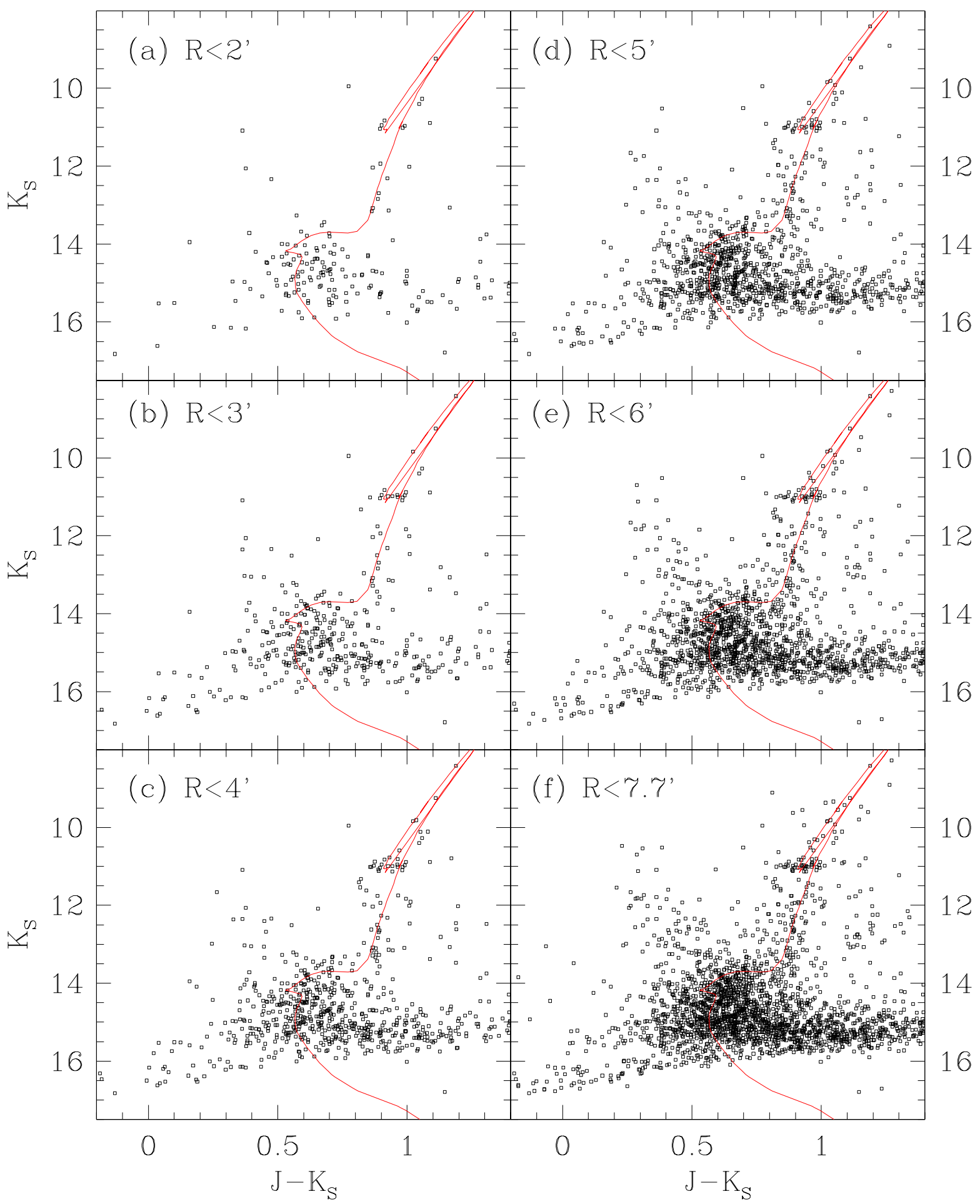

Fig. 9. - $K_{S}$ vs. $\left(J-K_{S}\right)$ CMDs for the stars in the field of $\operatorname{Tr} 5$. The solid lines represent the Padova isochrones (Bonatto, Bica, \& Girardi 2004) for $E(B-V)=0.67, K_{S}-M_{K_{S}}=12.66, \mathrm{Z}=0.008$, and age $=2.8 \mathrm{Gyr}$. 
$\left.E\left(J-K_{S}\right)=0.488 E(B-V)\right)$. The CMDs for the stars in the central regions (panels (a) and (b)) show best fits with the Padova isochrones. The CMDs, however, for stars in the intermediate regions (panels (c) and (d)) start to show hint of RGC tilt and RGB broadening, and those for stars in the outer regions (panels (e) and (f)) clearly show the effect of differential reddening (Piatti et al. 2004). The Padova isochrone fitting using the reddening value of $E(B-V)=0.60 \pm 0.10 \mathrm{mag}$ and the distance modulus obtained in the previous section $\left((m-M)_{0}=12.46 \pm 0.04 \mathrm{mag}\right)$ gives the best match with metallicity $[\mathrm{Fe} / \mathrm{H}]=-0.4 \pm 0.1$ dex and age $=2.8 \pm 0.2 \mathrm{Gyr}(\log t=9.45 \pm 0.04)$. Most of the recent studies after the year of 2000 give the age for $\mathrm{Tr}$ 5 between $\sim 3$ and 5 Gyr and the metallicity for $\operatorname{Tr} 5$ between $[\mathrm{Fe} / \mathrm{H}] \sim-0.30$ and -0.50 dex. This confirms that $\operatorname{Tr} 5$ is one of the oldest OCs in the Galaxy (see, e.g., Bonatto et al. 2006; Bonatto \& Bica 2007; Froebrich, Meusinger, \& Davis 2008; Momany et al. 2008; Froebrich et al. 2009), and that this cluster is a metalpoor OC not being a solar-metallicity cluster (Kaluzny 1998; Piatti et al. 2004).

In Figure 9, we have showed the Padova isochrone fittings for the $K_{S}$ vs $\left(J-K_{S}\right)$ CMDs, as in Figure 8 , in different radial regions as indicated in each panel from (a) $\mathrm{R}<2^{\prime}$ to (f) $\mathrm{R}<7.7^{\prime}$. Although all the same parameters are used for other parameters, somewhat larger reddening value $(E(B-V)=0.67 \pm 0.02)$ was used, because a poor fit is resulted from the reddening value of $E(B-V)=0.60$. This slight discrepancy seen in best-fit parameters among different diagrams might be caused by the uncertainties in the photometry and/or isochrones (Rider et al. 2004). We used the mean of the reddening values obtained from the Padova isochrone fittings for the $J$ vs. $(J-H)$ and $K_{S}$ vs $\left(J-K_{S}\right)$ CMDs, $<E(B-V)>=0.64 \pm 0.05$ in Table 1.

\section{COLOR-COLOR DIAGRAM}

Figure 10 shows the $(J-H) \times\left(H-K_{S}\right)$ color-color diagram of the stars with $K_{S}$ magnitude errors less than $0.1 \mathrm{mag}$ in $\operatorname{Tr} 5$ (filled circles). The MS range of the reddened Padova isochrones are overplotted as solid line $\left(0.15-1.4 M_{\odot}\right)$ and a reddening vector with $E(J-H)=1.72 \times E\left(H-K_{S}\right)$ for $A_{J}=0.55$ is denoted as an arrow. The locus of unreddened MS stars in the 2MASS system taken from Sung et al. (2008) is denoted by long-dashed line. The loci of unreddened MS and giants stars in the Bessell \& Brett (1988) system (not converted to the 2MASS system) taken from Bessell \& Brett (1988) are denoted by dotted and shortdashed lines, respectively.

Most of the stars in Tr 5 plotted in Figure 10 are distributed along the main sequences of the Padova isochrone, but in the locations affected by the reddenings of the amount of $A_{V}=1.98$ and $A_{J}=0.55$. The difference between the locus of the unreddened MS stars of Sung et al. (2008) and that of the reddened

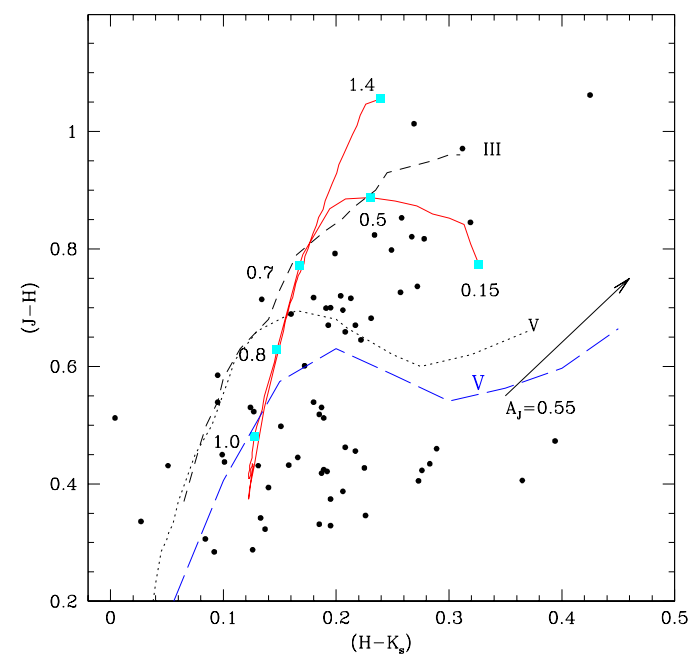

Fig. 10.- $(J-H) \times\left(H-K_{S}\right)$ color-color diagram of the stars in $\operatorname{Tr} 5$ for $K_{S}$ magnitude errors less than 0.1 mag (filled circles). The MS range of the reddened Padova isochrones are overplotted. Solid line is the MS range of the Padova isochrones of age $2.8 \mathrm{Gyr}$ and representative masses in $M_{\odot}$ are indicated along the isochrone. Long-dashed line is the locus of unreddened MS stars in the 2MASS system taken from Sung et al. (2008). Dotted and short-dashed lines are the loci of unreddened MS and giants stars, respectively, in the Bessell \& Brett (1988) system, not converted to the 2MASS system, taken from Bessell \& Brett (1988). Arrow is a reddening vector with $E(J-H)=1.72 \times E\left(H-K_{S}\right)$ for $A_{V}=1.98$ and $A_{J}=0.55$.

Padova isochrones is in very good agreement with the reddening value for Tr 5 denoted by the arrow. While Sung et al. (2008) have derived the mean locus of MS stars in the 2MASS system from compilation of 2MASS data for the late type MS stars in the solar neighborhood and various OCs, it would be highly anticipated to derive that for giant stars in the 2MASS system.

\section{SUMMARY}

We presented the analysis of the $J H K_{S}$ nearinfrared photometry for the old OC Tr 5 using the 2MASS data. We used these 2MASS $J H K_{S}$ photometry data for the study of $\operatorname{Tr} 5$ since this cluster is located very close to the Galactic plane $\left(b=+01 .^{\circ} 05\right)$ and has high $(E(B-V) \sim 0.6)$ interstellar reddening values. Since the $K$-band is relatively free of the differential reddening effect which exists in the face of $\mathrm{Tr}$ 5 , the distance to $\operatorname{Tr} 5$ is easily obtained using the $K$ band magnitude of the RGC of this cluster. The CMDs plotted for different radial regions clearly showed the differential reddening over the face of $\operatorname{Tr} 5$ (Piatti et 
al. 2004). The primary results obtained from the $J$ vs. $(J-H)$ and $K_{S}$ vs. $\left(J-K_{S}\right)$ CMDs of $\operatorname{Tr} 5$ are as follows :

1. The RGC stars are located at $J_{R G C}=11.9 \pm 0.1$, $K_{S, R G C}=11.0 \pm 0.1,(J-H)_{R G C}=0.73 \pm 0.03$, and $\left(J-K_{S}\right)_{R G C}=0.90 \pm 0.05$ and the distance to $\operatorname{Tr} 5$ is obtained $\left(\mathrm{d}=3.1 \pm 0.1 \mathrm{kpc},(m-M)_{0}=12.46 \pm 0.04\right)$ using the mean magnitude of the RGC stars in $K_{S^{-}}$ band.

2. From the Padova isochrone fittings to the CMDs, we have estimated the reddening, metallicity, and age : $E(B-V)=0.64 \pm 0.05,[\mathrm{Fe} / \mathrm{H}]=-0.4 \pm 0.1 \mathrm{dex}$, and age $=2.8 \pm 0.2$ Gyr $(\log t=9.45 \pm 0.04)$, respectively. These parameters confirm that $\operatorname{Tr} 5$ is an old OC with metallicity being metal-poorer than solar abundance, located in the anti-Galactic center region.

The authors are grateful to the anonymous referee for numerous comments and suggestions that improved the quality of this manuscript. This publication makes use of data products from the Two Micron All Sky Survey, which is a joint project of the University of Massachusetts and the Infrared Processing and Analysis Center/California Institute of Technology, funded by the National Aeronautics and Space Administration. This research has made use of the SIMBAD database, operated at CDS, Strasbourg, France.

\section{REFERENCES}

Ahumada, J. \& Lapasset, E., 1995, Catalogue of blue stragglers in open clusters, A\&AS, 109, 375

Ahumada, J. A. \& Lapasset, E., 2007, New catalogue of blue stragglers in open clusters, A\&A, 463, 789

Alves, D. R., 2000, K-band calibration of the red clump luminosity, ApJ, 539, 732

Bessell, M. S. \& Brett, J. M., 1988, JHKLM photometry - Standard systems, passbands, and intrinsic colors, PASP, 100, 1134

Bica, E., Bonatto, C., \& Blumberg, R., 2006, Faint open clusters with 2MASS: BH63, Lyngå 2, Lyngå 12 and King 20, A\&A, 460, 83

Bica, E., Dutra, C. M., \& Barbuy, B., 2003, A catalogue of infrared star clusters and stellar groups, A\&A, 397,177

Bonatto, C. J., Bica, E., \& Girardi, L., 2004, Theoretical isochrones compared to 2MASS observations: Open clusters at nearly solar metallicity, A\&A, 415, 571

Bonatto, C., Bica, E., \& Santos, J. F. C., Jr., 2005, Spatial dependence of 2MASS luminosity and mass functions in the old open cluster NGC 188, A\&A, 433, 917

Bonatto, C., Kerber, L. O., Bica, E., \& Santiago, B. X., 2006, Probing disk properties with open clusters, A\&A, 446, 121
Bonatto, C. \& Bica, E., 2007, Old open clusters in the inner Galaxy: FSR 1744, FSR 89 and FSR 31, A\&A, 473,445

Cardelli, J. A., Clayton, G. C., \& Mathis, J. S., 1989, The relationship between infrared, optical, and ultraviolet extinction, ApJ, 345, 245-256

Carpenter, J. M., 2001, Color transformations for the 2MASS second incremental data release, AJ, 121, 2851

Carraro, G., Vázquez, R. A., \& Moitinho, A., 2008, Blue straggler stars in Galactic open clusters and the effect of field star contamination, A\&A, 482, 777

Carrera, R., Gallart, C., Pancino, E., \& Zinn, R., 2007, The Infrared Ca II triplet as metallicity indicator, AJ, 134, 1298

Chen, L., Hou, J. L., \& Wang, J. J., 2003, On the Galactic disk metallicity distribution from open clusters. I. New catalogs and abundance gradient, AJ, 125,1397

Cole, A. A., Smecker-Hane, T. A., Tolstoy, E., Bosler, T. L., \& Gallagher, J. S., 2004, The effects of age on red giant metallicities derived from the near-infrared Ca II triplet, MNRAS, 347, 367

De Marchi, F., De Angeli, F., Piotto, G., Carraro, G., \& Davies, M. B., 2006, Search and analysis of blue straggler stars in open clusters, A\&A, 459, 489

Dias, W. S., Alessi, B. S., Moitinho, A., \& Lepine, J. R. D., 2002, New catalogue of optically visible open clusters and candidates, A\&A, 389, 871

Dow, M. J. \& Hawarden, T. G., 1970, The open cluster Trumpler 5, M. N. A. S. So. Africa, 29, 137

Friel, E. D., 1995, The old open clusters of the Milky Way, ARA\&A, 33, 381

Froebrich, D., Scholz, A., \& Raftery, C. L., 2007, A systematic survey for infrared star clusters with $|b|<$ $20^{\circ}$ using 2MASS, MNRAS, 374, 399

Froebrich, D., Meusinger, H., \& Davis, C. J., 2008, FSR 0190: another old distant Galactic cluster, MNRAS, 383, L45

Froebrich, D., Meusinger, H., Davis, C. J., \& Schmeja, S., 2009, UKIRT follow-up observations of the old open cluster FSR 0358 (Kirkpatrick1), MNRAS, 395, 1768

Grocholski, A. J. \& Sarajedini, A., 2002, WIYN open cluster study. X. The K-band magnitude of the red clump as a distance indicator, AJ, 123, 1603

Janes, K. \& Adler, D., 1982, Open clusters and Galactic structure, ApJS, 49, 425

Janes, K. A. \& Phelps, R. L., 1994, The galactic system of old star clusters: The development of the galactic disk, AJ, 108, 1773

Kalinowski, J. K., 1974, Observations of the carbon star V493 Monocerotis, IAU Info. Bull. on Var. Stars, No. 924 
Kalinowski, J. K., 1975, The open cluster Trumpler 5, BAAS, 7, 542

Kalinowski, J. K., 1979, The old disk cluster Trumpler 5, Ph.D. thesis, Indiana University

Kalinowski, J. K., Burkhead, M. S., \& Honeycutt, R. K., 1974, On a possible carbon-star member of the old open cluster Trumpler 5, ApJ, 193, L77

Kaluzny, J., 1998, CCD photometry of distant open clusters IV. Trumpler 5, A\&AS, 133, 25

Kharchenko, N. V., Piskunov, A. E., Röser, S., Schilbach, E., \& Scholz, R.-D., 2005, 109 new Galactic open clusters, A\&A, 440, 403

Kim, S. C., 2006, Near-infrared photometric study of the Galactic open clusters NGC 1641 and NGC 2394 based on 2MASS data, JKAS, 39, 115

Kim, S. C. \& Sung, H., 2003, Physical parameters of the old open cluster Trumpler 5, JKAS, 36, 13

Kronberger, M., Teutsch, P., Alessi, B., Steine, M., Ferrero, L., Graczewski, K., Juchert, M., Patchick, D., Riddle, D., Saloranta, J., Schoenball, M., \& Watson, C., 2006, New Galactic open cluster candidates from DSS and 2MASS imagery, A\&A, 447, 921

Kyeong, J., Kim, S. C., Hiriart, D., \& Sung, E.-C., 2008, UBVI CCD photometry of the old open cluster NGC 1193, JKAS, 41, 147

Lada, C. J., \& Lada, E. A., 2003, Embedded clusters in molecular clouds, ARA\&A, 41, 57

Lyngå, G., 1987, "Catalogue of Open Cluster Data" (Observatoire de Strassbourg, Centre de Données Stellaires)

Momany, Y., Ortolani, S., Bonatto, C., Bica, E., \& Barbuy, B., 2008, Multi-conjugate Adaptive optics VLT imaging of the distant old open cluster FSR 1415, MNRAS, 391, 1650

Paczyński, B., \& Stanek, K. Z. 1998, Galactocentric distance with the optical gravitational lensing experiment and HIPPARCOS red clump stars, ApJ, 494, L219

Paunzen, E. \& Netopil, M., 2006, On the current status of open-cluster parameters, MNRAS, 371, 1641

Piccirillo, J., Kalinowski, J. K., \& Wing, R. F., 1977, The red giants of Trumpler 5, BAAS, 9, 637

Piatti, A. E., Clariá, J. J., \& Ahumada, A. V., 2004, The old open cluster Trumpler 5: a reddened, metalpoor anticentre cluster, MNRAS, 349, 641

Rider, C. J., Tucker, D. L., Smith, J. A., Stoughton, C., Allam, S. S., \& Neilsen, E. H., 2004, A survey of open clusters in the u'g'r'i'z' filter system: I. Results for NGC 2548 (M48), AJ, 127, 2210

Salaris, M., Weiss, A., \& Percival, S. M., 2004, The age of the oldest Open Clusters, A\&A, 414, 163
Skrutskie, M. F., Schneider, S. E., Stiening, R., et al., 1997, The Two Micron All Sky Survey (2MASS): Overview and Status, in The Impact of Large Scale Near-IR Sky Surveys, eds. F. Garzón, N. Epchtein, A. Omont, B. Burton and P. Persi, Kluwer (Netherlands), 25

Skrutskie, M. F., Cutri, R. M., Stiening, R., et al., 2006, The Two Micron All Sky Survey (2MASS), AJ, 131, 1163

Stanek, K. Z. \& Garnavich, P. M., 1998, Distance to M31 with the Hubble Space Telescope and HIPPARCOS red clump stars, ApJ, 503, L131

Sung, H., Bessell, M. S., Chun, M.-Y., Karimov, R., \& Ibrahimov, M., 2008, The initial mass function and young brown dwarf candidates in NGC 2264 . III. Photometric data, AJ, 135, 441

Sung, H., Stauffer, J. R., \& Bessell, M. S., 2009, A Spitzer view of the young open cluster NGC 2264, AJ, 138, 1116 